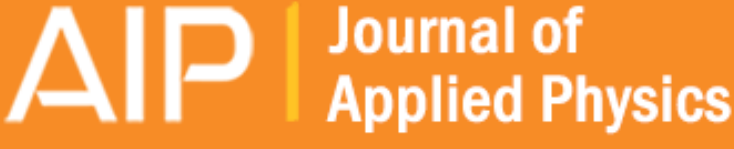

\section{Fano interference and a slight fluctuation of the Majorana hallmark}

A. C. Seridonio, E. C. Siqueira, F. A. Dessotti, R. S. Machado, and M. Yoshida

Citation: Journal of Applied Physics 115, 063706 (2014); doi: 10.1063/1.4865503

View online: http://dx.doi.org/10.1063/1.4865503

View Table of Contents: http://scitation.aip.org/content/aip/journal/jap/115/6?ver=pdfcov

Published by the AIP Publishing

\section{Articles you may be interested in}

Quantum transport through the system of parallel quantum dots with Majorana bound states

J. Appl. Phys. 115, 083706 (2014); 10.1063/1.4867040

Identifying Dirac cones in carbon allotropes with square symmetry

J. Chem. Phys. 139, 184701 (2013); 10.1063/1.4828861

Tunneling transport through multi-quantum-dot with Majorana bound states

J. Appl. Phys. 114, 033703 (2013); 10.1063/1.4813229

Signature of quantum interference and the Fano resonances in the transmission spectrum of bilayer graphene nanostructure

J. Appl. Phys. 110, 014306 (2011); 10.1063/1.3603005

Simultaneous current-, force-, and work-function measurement with atomic resolution

Appl. Phys. Lett. 86, 153101 (2005); 10.1063/1.1900316

\section{AIP $\mid$ chaos CALL FOR APPLICANTS Seeking new Editor-in-Chief}




\title{
Fano interference and a slight fluctuation of the Majorana hallmark
}

\author{
A. C. Seridonio, ${ }^{1,2}$ E. C. Siqueira, ${ }^{2}$ F. A. Dessotti, ${ }^{2}$ R. S. Machado, ${ }^{2}$ and M. Yoshida ${ }^{1}$ \\ ${ }^{1}$ Instituto de Geociências e Ciências Exatas-IGCE, Universidade Estadual Paulista, Departamento de Física, \\ 13506-970, Rio Claro, São Paulo, Brazil \\ ${ }^{2}$ Departamento de Física e Química, Universidade Estadual Paulista, 15385-000, Ilha Solteira, \\ São Paulo, Brazil
}

(Received 19 December 2013; accepted 31 January 2014; published online 13 February 2014)

\begin{abstract}
According to the Liu and Baranger [Phys. Rev. B 84, 201308(R) (2011)], an isolated Majorana state bound to one edge of a long enough Kitaev chain in the topological phase and connected to a quantum dot, results in a robust transmittance of $1 / 2$ at zero-bias. In this work, we show that the removal of such a hallmark can be achieved by using a metallic surface hosting two adatoms in a scenario where there is a lack of symmetry in the Fano effect, which is feasible by coupling the Kitaev chain to one of these adatoms. Thus in order to detect this feature experimentally, one should apply the following two-stage procedure: (i) first, attached to the adatoms, one has to lock AFM tips in opposite gate voltages (symmetric detuning of the levels $\Delta \varepsilon$ ) and measure by an STM tip, the zero-bias conductance; (ii) thereafter, the measurement of the conductance is repeated with the gates swapped. For $|\Delta \varepsilon|$ away from the Fermi energy and in the case of strong coupling tip-host, this approach reveals in the transmittance, a persistent dip placed at zero-bias and immune to the aforementioned permutation, but characterized by an amplitude that fluctuates slightly around 1/2. However, in the case of a tip acting as a probe, the adatom decoupled from the Kitaev chain becomes completely inert and no fluctuation is observed. Therefore, the STM tip must be considered in the same footing as the "host+adatoms" system. As a result, we have found that despite the small difference between these two Majorana dips, the zero-bias transmittance as a function of the symmetric detuning yields two distinct behaviors, in which one of them is unpredictable by the standard Fano's theory. Therefore, to access such a non trivial pattern of Fano interference, the hypothesis of the STM tip acting as a probe should be discarded. C 2014 AIP Publishing LLC. [http://dx.doi.org/10.1063/1.4865503]
\end{abstract}

\section{INTRODUCTION}

Majorana fermions are particles that constitute their own antiparticles. Such a proposal was made almost a century ago by Ettore Majorana in the context of high-energy physics. In solid state systems, these exotic particles are not fundamental but emerge as quasiparticle excitations. ${ }^{1}$ This species of excitation is ranked as non-Abelian anyons and obeys an unusual quantum statistics. Its most remarkable property lies on the possibility of bounding two far apart Majoranas that define an unique nonlocal Dirac fermion. Once this spatially delocalized state is occupied, it yields a robust qubit decoupled from the surroundings, thus avoiding decoherence due to perturbations. This protected qubit then enlarges the feasibility to make these blocks as essential to the accomplishment of a topological quantum computer. Thus, in the last few years, the quest for devices nesting Majorana fermions has received much attention from the community of researchers working with quantum computing. ${ }^{2-6}$

To the best knowledge, the superconductor state is considered suitable for the emergence of Majorana excitations. Superconductivity lies on Cooper-pair condensation and spontaneous breaking of charge conservation, thus leading to the superposition of electrons and holes. However, s-wave superconductivity arises from electrons with opposite spins that result in distinct operators for creation and annihilation of quasiparticles, thus preventing the realization of Majorana bound states (MBSs). To support them, a spinless superconductor is indeed required. Such conditions can be found in the topological phase of the Kitaev chain, ${ }^{7}$ which offers the proper environment to sustain Majoranas. The Majoranas are zero-energy modes, in particular, placed at the edges of this chain.

The engineering of a sample with $p$-wave superconductivity can be achieved experimentally by proximity effect. It is known that a $s$-wave superconductor nearby a semiconducting nanowire with strong spin-orbit interaction and crossed by a magnetic field, induces $p$-wave superconductivity on the latter system. ${ }^{8-16}$ Additionally, the existence of Majoranas are predicted in the fractional quantum Hall state with filling factor $\nu=5 / 2,{ }^{17}$ in three-dimensional topological insulators ${ }^{18}$ and at the core of superconducting vortices. ${ }^{19-21}$ In this scenario, quantum transport becomes a sensible tool for detecting Majorana quasiparticles. Particularly in Ref. 22, it was predicted for the experimental setup of a single quantum dot (QD) side-coupled to a Majorana state, that the zerobias peak (ZBP) for the conductance should be given by the robust Majorana hallmark $\mathcal{G}=0.5 \mathcal{G}_{0}$, where $\mathcal{G}_{0}=e^{2} / h$ is the background conductance. We highlight that in Ref. 23, Vernek et al. have determined that such an amplitude arises from the leaking of the Majorana state into the QD.

Experimentally, a persistent ZBP has been observed in transport measurements through a setup composed by a nanowire of indium antimonide merged to gold and niobium titanium nitride. ${ }^{24}$ In this aforementioned system, Majoranas are 
supposed to exist due to the ZBP that stands up to a wide range of magnetic fields and gate voltages. Such a robustness of the ZBP has also been found in the analogous system of a superconductor of aluminium close to a nanowire of indium arsenide. ${ }^{25}$ Moreover, we stress that the ZBP feature may also have another physical origin, for instance, the Kondo effect. $^{26-33}$

In this context, an apparatus based on Fano effect ${ }^{34,35}$ becomes an alternative approach to detect a Majorana state. Here, we benefit of this mechanism, an interference phenomenon found in systems where tunneling channels compete for the electron transport. This effect can be detectable by the Scanning Tunneling Microscope (STM), a device made by a metallic tip that detects, for low enough temperatures, the transmittance through a system by measuring the differential conductance. $^{29-33}$ Thus, we have studied theoretically the conductance probed by an STM tip of a metallic surface coupled to two adatoms, in which one of them is coupled to a MBS hosted by a long enough Kitaev chain in the topological phase. We should remark that nowadays such a chain is achievable experimentally as found in Ref. 24, whose system becomes the most promising candidate to our proposal [see Fig. 1].

Additionally, we have considered in the model two Atomic Force Microscope (AFM) tips capacitively coupled to the adatoms, just in order to tune their levels as proposed in Ref. 36. Our approach employs the spinless Hamiltonian of Ref. 22 in combination with the equation-of-motion procedure for the Green's functions.

(a) Top view:

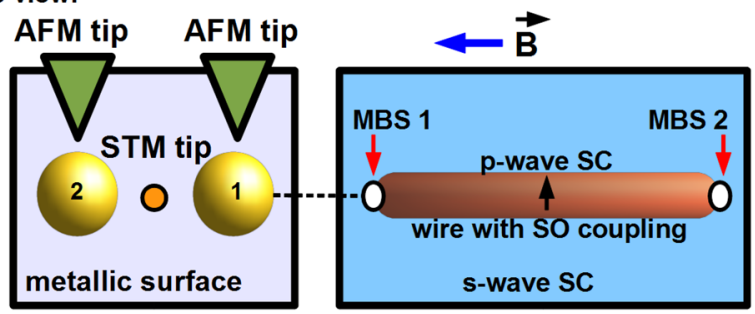

(b) Front view:

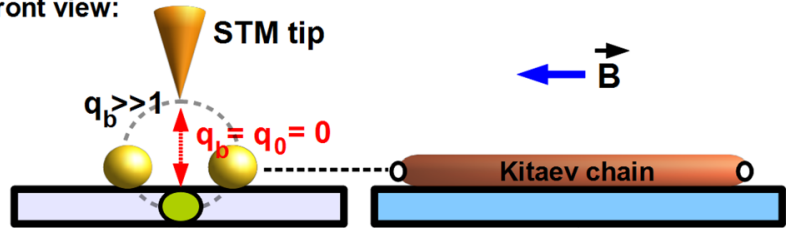

FIG. 1. Two perspectives of the same apparatus: in panel (a) we have the top view, while (b) represents the front view. In both, Majorana bound states (MBSs) appear lying on a long enough Kitaev chain within the topological phase [right side of panels (a) and (b)], which can be accomplished as proposed experimentally in Ref. 24: s-wave superconductivity (SC) inducing pwave pairing in a semiconducting wire with strong spin-orbit interaction (SO) and crossed by a perpendicular magnetic field $\vec{B}$. Here we follow such a proposal by adding an STM tip nearby a metallic surface coupled to two adatoms, in which one of them is hybridized with a MBS 1 (a half-electron state). The MBS 1 is connected far apart to a MBS 2. The AFM tips are employed to tune the levels of the adatoms. This device explores the lack of symmetry in Fano interference, which is detectable via the zero-bias conductance. The parameters $q_{0}$ and $q_{b}$ are Fano factors due to the interference between the different paths taken by the electrons from the tip to the surface. When $q_{b} \gg 1$ the hybridization between the tip and the adatoms is stronger than the hybridization to the surface. In this case the electrons tunnel to the surface throughout the adatoms. In contrast, for $q_{0}=0$ the electrons tunnel directly to the surface. The green-circle represents the site of the host side-coupled to the adatoms.
By determining the transmittance of this setup, we have found Fano profiles due to the coupling between the setup of the adatoms and an isolated MBS. For the setup decoupled from this MBS, the direct and the mixed Green's functions are symmetric with respect to the labels 1 and 2 that designate the parameters of the adatoms. In the opposite limit, this symmetry property is broken and the swap of the indexes $1 \leftrightarrow 2$ leads to a lack of symmetry in the Fano profile.

This lack of symmetry can be accessed experimentally by performing the following proposed two-stage procedure: (i) first, attached to the adatoms, one has to lock AFM tips in opposite gate voltages (symmetric detuning of the levels $\Delta \varepsilon$ ) and measure by an STM tip, the zero-bias conductance; (ii) thereafter, the measurement of the conductance is repeated with the gates swapped.

As a result of this method and the Fano regime as well, the transmittance for $|\Delta \varepsilon|$ away from the Fermi energy exhibits a zero-bias dip persistent against the permutation of the gate voltages. For the case in which the STM acts as a probe of the LDOS (local density of states) for the "host+adatoms" system, the adatom decoupled from the Kitaev chain plays no role and the typical Majorana hallmark is verified: a robust zero-bias transmittance characterized by an amplitude of $1 / 2$ as that found in Ref. 22 for a single QD setup. On the other hand, for the STM in the same footing as the "host + adatoms" system, a slight fluctuation around the amplitude of 1/2 manifests as a straight aftermath of the two-stage procedure in combination with the adatom free of the MBS. However, despite the small difference between these two Majorana dips, each one leads to a particular Fano lineshape for the zero-bias transmittance as a function of the symmetric detuning. Therefore, we demonstrate in this work that the assumption of the STM as a probe tip is not enough to reveal the unexpected pattern of Fano interference for the proposed setup of Fig. 1.

This paper is organized as follows. In Sec. II, we show the theoretical model for the system sketched in Fig. 1 as well as the derivation of the transmittance. The Green's functions of the adatoms are also presented in this section. The results appear in Sec. III and in Sec. IV, we summarize the conclusions.

\section{THEORETICAL MODEL}

\section{A. Hamiltonian}

The system we investigate is described according to the Hamiltonian

$$
\mathcal{H}_{\text {total }}=\mathcal{H}_{\text {host }+ \text { ads }}+\mathcal{H}_{\text {tip }}+\mathcal{H}_{\text {tun }}
$$

In order to mimic the system outlined in Fig. 1, we follow the spinless Hamiltonian proposed by Liu et al., ${ }^{22}$ taking two adatoms into account, which reads

$$
\begin{aligned}
\mathcal{H}_{\text {host }+ \text { ads }}= & \sum_{k}\left(\varepsilon_{k}-\mu_{\text {host }}\right) c_{k}^{\dagger} c_{k}+\sum_{j} \varepsilon_{j} d_{j}^{\dagger} d_{j} \\
& +V\left(\sum_{j k} c_{k}^{\dagger} d_{j}+\text { H.c. }\right)+i \epsilon_{M} \eta_{1} \eta_{2} \\
& +\lambda\left(d_{1}-d_{1}^{\dagger}\right) \eta_{1},
\end{aligned}
$$


where the electrons in the host are described by the operator $c_{k}^{\dagger}\left(c_{k}\right)$ for the creation (annihilation) of an electron in a quantum state labeled by the wave number $k$, energy $\varepsilon_{k}$ and chemical potential $\mu_{\text {host }}$. For the adatoms, $d_{j}^{\dagger}\left(d_{j}\right)$ creates (annihilates) an electron in the state $\varepsilon_{j}$, with $j=1,2 . V$ is the hybridization of the adatoms with the host. In particular for $j=1$, the adatom 1 is coupled to the MBS 1 described by the operator $\eta_{1}^{\dagger}=\eta_{1}$. The strength of this coupling is $\lambda$. The MBS 2 given by $\eta_{2}^{\dagger}=\eta_{2}$ is connected to the MBS 1 via the coefficient $\epsilon_{M} \sim e^{-L / \xi}$, with $L$ being the distance between the MBSs and $\xi$ the coherence length. It is worth mentioning that the present spinless model supposes a strong magnetic field over the entire system, which leads to a large Zeeman splitting where the higher levels are not energetic favorable at low temperatures. In this situation, one spin component plays no role and the spin degrees of freedom can be ignored.

The second part of Eq. (1) is described by the Hamiltonian

$$
\mathcal{H}_{\text {tip }}=\sum_{q}\left(\varepsilon_{q}-\mu_{\text {tip }}\right) b_{q}^{\dagger} b_{q}
$$

which corresponds to free electrons ruled by fermionic operators $b_{q}^{\dagger}$ and $b_{q}$ in the STM tip, with energy $\varepsilon_{q}$ and chemical potential $\mu_{\text {tip. }}$.

To perform the coupling between Eqs. (2) and (3), we have to define the tunneling Hamiltonian

$$
\mathcal{H}_{\text {tun }}=w\left(f_{t}^{\dagger} \psi_{0}+\text { H.c. }\right)
$$

where $w$ is the STM tip-host coupling,

$$
f_{t}=\sum_{q} b_{q}
$$

is for the edge of the STM tip,

$$
\psi_{0}=f_{0}+\left(\pi \Gamma \rho_{0}\right)^{1 / 2} q_{0} \sum_{j} d_{j}
$$

is the field operator that accounts for Fano interference,

$$
f_{0}=\sum_{k} c_{k}
$$

represents the host site laterally coupled to the adatoms [see the green-circle of the host outlined in Fig. 1],

$$
\Gamma=\pi V^{2} \rho_{0}
$$

is the Anderson parameter, with $\rho_{0}=\frac{1}{2 D}$ as the density of states for the surface without adatoms, $D$ is the band halfwidth and

$$
q_{0}=\left(\pi \Gamma \rho_{0}\right)^{-1 / 2}\left(\frac{\tilde{V}}{w}\right)
$$

is the Fano factor of interference, ${ }^{37}$ with $\tilde{V}$ as the couplings between the STM tip and the adatoms. Notice that due to Eqs. (6) and (9), the limit $q_{0} \gg 1$ represents the situation in which the tip is highly hybridized with the adatoms, while in the opposite regime $q_{0}=0$, the tip is strongly connected to the surface [see Fig. 1]. As the former case in presence of a MBS still obeys the standard Fano's theory, in this work we will focus on the latter, where we can find a non trivial Fano interference. Such a point will be discussed in Sec. III.

\section{B. Calculation of the transmittance}

\section{The STM tip as a probe}

By applying the linear response theory, in which the STM tip is considered as a probe, it is possible to show that the zero-bias conductance is given by

$$
\mathcal{G}(0)=\frac{e^{2}}{h}(2 \pi w)^{2} \int \rho_{\text {LDOS }}(\varepsilon) \rho_{\text {tip }}(\varepsilon)\left(-\frac{\partial f_{F}}{\partial \varepsilon}\right) d \varepsilon,
$$

where $e$ is the electron charge, $h$ is the Planck constant, $\rho_{\text {LDOS }}(\varepsilon)$ is the LDOS of the "host+adatoms" system, $\rho_{\text {tip }}$ $(\varepsilon)$ as the DOS of the STM tip and $f_{F}$ is the Fermi-Dirac distribution. The total transmittance is then defined as follows:

$$
\mathcal{T}_{\text {probe }}(\varepsilon)=(2 \pi w)^{2} \rho_{\text {LDOS }}(\varepsilon) \rho_{\text {tip }}(\varepsilon)
$$

To obtain the LDOS, we follow Ref. 36 by introducing the retarded Green's function

$$
\mathcal{R}_{\psi_{0} \psi_{0}}=-\frac{i}{\hbar} \theta(t) \operatorname{Tr}\left\{\varrho_{\text {host }+ \text { ads }}\left[\psi_{0}(t), \psi_{0}^{\dagger}(0)\right]_{+}\right\}
$$

for the field operator of Eq. (6) in the time domain $t$, where $\theta(t)$ is the Heaviside function, $\varrho_{\text {host+ads }}$ is the density matrix of the system described by the Hamiltonian in Eq. (2) and $[\cdots, \cdots]_{+}$is the anticommutator of Eq. (6) at distinct times. From Eq. (12), the LDOS of the host can be obtained as

$$
\rho_{\mathrm{LDOS}}(\varepsilon)=-\frac{1}{\pi} \operatorname{Im}\left(\tilde{\mathcal{R}}_{\psi_{0} \psi_{0}}\right),
$$

where $\tilde{\mathcal{R}}_{\psi_{0} \psi_{0}}$ is the Fourier transform of $\mathcal{R}_{\psi_{0} \psi_{0}}$ in the energy domain $\varepsilon$. Analogously, we have

$$
\rho_{\text {tip }}(\varepsilon)=-\frac{1}{\pi} \operatorname{Im}\left(\tilde{\mathcal{R}}_{f_{t} f_{t}}\right)
$$

with

$$
\mathcal{R}_{f_{t} f_{t}}=-\frac{i}{\hbar} \theta(t) \operatorname{Tr}\left\{\varrho_{\text {tip }}\left[f_{t}(t), f_{t}^{\dagger}(0)\right]_{+}\right\},
$$

where $\varrho_{\text {tip }}$ is the density matrix of the system described by the Hamiltonian in Eq. (3).

Thus to determine an analytical expression for the LDOS, we apply the equation-of-motion approach on Eq. (12). Such a procedure is summarized as follows:

$$
(\varepsilon+i \eta) \tilde{\mathcal{R}}_{\mathcal{A B}}=\left[\mathcal{A}, \mathcal{B}^{\dagger}\right]_{+}+\tilde{\mathcal{R}}_{\left[\mathcal{A}, \mathcal{H}_{i}\right] \mathcal{B}}
$$

with $\eta \rightarrow 0^{+}, \mathcal{A}$ and $\mathcal{B}$ as fermionic operators belonging to the Hamiltonian $\mathcal{H}_{i}(i=$ host + ads or tip $)$. 
By taking Eq. (12), one can calculate via Eqs. (2), (6), and (16) with $\mathcal{A}=\mathcal{B}=\psi_{0}$ and $\mathcal{H}_{i}=\mathcal{H}_{\text {host+ads }}$, the following relation:

$$
\begin{aligned}
\tilde{\mathcal{R}}_{\psi_{0} \psi_{0}}= & \tilde{\mathcal{R}}_{f_{0} f_{0}}+\left(\pi \Gamma \rho_{0}\right) q_{0}^{2} \sum_{j l} \tilde{\mathcal{R}}_{d_{j} d_{l}}+2\left(\pi \Gamma \rho_{0}\right)^{1 / 2} q_{0} \\
& \times \sum_{j} \tilde{\mathcal{R}}_{d_{j} f_{0}},
\end{aligned}
$$

which depends on the Green's functions $\tilde{\mathcal{R}}_{f_{0} f_{0}}, \tilde{\mathcal{R}}_{f_{0} d_{j}}$ and $\tilde{\mathcal{R}}_{d_{j} d_{l}}$. First, we find $\tilde{\mathcal{R}}_{f_{0} f_{0}}$,

$$
\tilde{\mathcal{R}}_{f_{0} f_{0}}=\pi \rho_{0}(\bar{\gamma}-i)+\pi \rho_{0} \Gamma(\bar{\gamma}-i)^{2} \sum_{j l} \tilde{\mathcal{R}}_{d_{j} d_{l}}
$$

and later on, the mixed Green's function $\tilde{\mathcal{R}}_{d_{j} f_{0}}$,

$$
\tilde{\mathcal{R}}_{d_{j} f_{0}}=\sqrt{\pi \Gamma \rho_{0}}(\bar{\gamma}-i) \sum_{l} \tilde{\mathcal{R}}_{d_{j} d_{l}},
$$

where

$$
\bar{\gamma}=\frac{1}{\pi \rho_{0}} \sum_{k} \frac{1}{\varepsilon-\varepsilon_{k}} .
$$

Now, we choose for Eq. (16), $\mathcal{H}_{i}=\mathcal{H}_{\text {tip }}$ and $\mathcal{A}=\mathcal{B}=f_{t}$, respectively, from Eqs. (3) and (14), to show that

$$
\tilde{\mathcal{R}}_{f_{t} f_{t}}=\pi \rho_{0}(\bar{\gamma}-i) .
$$

In particular, for the wide band limit $D \rightarrow \infty, \bar{\gamma} \rightarrow 0$. Thus, the imaginary parts of Eqs. (18)-(21) become

$$
\begin{gathered}
\operatorname{Im}\left(\tilde{\mathcal{R}}_{f_{0} f_{0}}\right)=-\pi \rho_{0}\left[1+\Gamma \sum_{j l} \operatorname{Im}\left(\tilde{\mathcal{R}}_{d_{j} d_{l}}\right)\right], \\
\operatorname{Im}\left(\tilde{\mathcal{R}}_{d_{j} f_{0}}\right)=-\sqrt{\pi \Gamma \rho_{0}} \sum_{l} \operatorname{Re}\left(\tilde{\mathcal{R}}_{d_{j} d_{l}}\right)
\end{gathered}
$$

and

$$
\operatorname{Im}\left(\tilde{\mathcal{R}}_{f_{t} f_{t}}\right)=-\pi \rho_{0} .
$$

Now, we take Eqs. (22)-(24) into Eq. (11) to obtain

$$
\begin{aligned}
T_{\text {probe }}(\varepsilon)=\frac{\mathcal{T}_{\text {probe }}(\varepsilon)}{\mathcal{T}_{b}}= & 1+\Gamma \sum_{j l}\left[\left(1-q_{0}^{2}\right) \operatorname{Im}\left(\tilde{\mathcal{R}}_{d_{j} d_{l}}\right)\right. \\
& \left.+2 q_{0} \operatorname{Re}\left(\tilde{\mathcal{R}}_{d_{j} d_{l}}\right)\right]
\end{aligned}
$$

as the total transmittance through the system, expressed in terms of the background conductance

$$
\mathcal{T}_{b}=4 x=4\left(\pi w \rho_{0}\right)^{2},
$$

and the Green's functions $\tilde{\mathcal{R}}_{d_{j} d_{l}}$ of the adatoms.

\section{The STM tip in the same footing as the "host+adatoms" system}

Here, we derive the Landauer-Büttiker formula for the zero-bias conductance $\mathcal{G}(0)$ by considering the STM tip in the same footing as the "host+adatoms" system, which is achievable with $\tilde{V}=V$ in Eq. (9).

The zero-bias conductance is a function of the transmittance $\mathcal{T}_{\text {full }}(\varepsilon)$ as follows:

$$
\mathcal{G}(0)=\frac{\partial}{\partial \varphi} \mathcal{J}_{\text {host }}(\varphi=0)=\frac{e^{2}}{h} \int d \varepsilon\left(-\frac{\partial f_{F}}{\partial \varepsilon}\right) \mathcal{T}_{\text {full }}(\varepsilon),
$$

with $\mathcal{J}_{\text {host }}$ as the current for the host and $\mu_{\text {host }}-\mu_{\text {tip }}=e \varphi$, with $\varphi$ as the applied bias-voltage. We begin with the transformation

$$
\left(\begin{array}{l}
c_{k} \\
b_{k}
\end{array}\right)=\left(\begin{array}{cc}
\frac{1}{\sqrt{2}} & \frac{1}{\sqrt{2}} \\
-\frac{1}{\sqrt{2}} & \frac{1}{\sqrt{2}}
\end{array}\right)\left(\begin{array}{c}
c_{o k} \\
c_{e k}
\end{array}\right),
$$

on the Hamiltonian of Eq. (1), which depends on the even and odd conduction operators $c_{e k}$ and $c_{o k}$, respectively. These definitions allow us to express Eq. (1) as

$$
\mathcal{H}=\mathcal{H}_{e}+\mathcal{H}_{o}+\tilde{\mathcal{H}}_{\text {tun }}=\mathcal{H}_{\varphi=0}+\tilde{\mathcal{H}}_{\text {tun }},
$$

where

$$
\begin{aligned}
\mathcal{H}_{e}= & \sum_{k} \varepsilon_{k} c_{e k}^{\dagger} c_{e k}+\sum_{j} \varepsilon_{j} d_{j}^{\dagger} d_{j} \\
& +\sum_{j k} \sqrt{2} V\left(c_{e k}^{\dagger} d_{j}+\text { H.c. }\right)+w \sum_{k q} c_{e k}^{\dagger} c_{e q} \\
& +i \epsilon_{M} \eta_{1} \eta_{2}+\lambda\left(d_{1}-d_{1}^{\dagger}\right) \eta_{1}
\end{aligned}
$$

represents the Hamiltonian part of the system coupled to the adatoms via an effective hybridization $\sqrt{2} V$, while

$$
\mathcal{H}_{o}=\sum_{k} \varepsilon_{k} c_{o k}^{\dagger} c_{o k}-w \sum_{k q} c_{o k}^{\dagger} c_{o q}
$$

is the decoupled one. However, they are connected to each other by the tunneling Hamiltonian

$$
\tilde{\mathcal{H}}_{\text {tun }}=-\Delta \mu \sum_{k}\left(c_{e k}^{\dagger} c_{o k}+c_{o k}^{\dagger} c_{e k}\right),
$$

with $\mu_{\text {host }}=\Delta \mu, \mu_{\text {tip }}=-\Delta \mu$ and $\Delta \mu=e \varphi / 2$. As in the zero-bias regime $\Delta \mu \rightarrow 0$, due to $\varphi \rightarrow 0, \tilde{\mathcal{H}}_{\text {tun }}$ is a perturbative term.

Here, we use the interaction picture to calculate $\mathcal{T}_{\text {full }}(\varepsilon)$. It ensures that a state $\left|\Phi_{n}\right\rangle$ from the spectrum of the Hamiltonian given by Eq. (29) admits the following timedependency:

$$
\begin{aligned}
\left|\Phi_{n}\right\rangle & =e^{-\frac{i}{\hbar} \int_{-\infty}^{0} \tilde{\mathcal{H}}_{\text {tun }}(\tau) d \tau}\left|\Psi_{n}\right\rangle \\
& \simeq\left(1-\frac{i}{\hbar} \int_{-\infty}^{0} \tilde{\mathcal{H}}_{\text {tun }}(\tau) d \tau\right)\left|\Psi_{n}\right\rangle,
\end{aligned}
$$

where $\hbar=\frac{h}{2 \pi}$ and $\left|\Psi_{n}\right\rangle$ is an eigenstate of $\mathcal{H}_{e}+\mathcal{H}_{o}=\mathcal{H}_{\varphi=0}$. Thus, the current $\mathcal{J}_{\text {host }}$ for the host can be obtained by 
performing the expected mean value of the current operator $\mathcal{I}_{\text {host }} \equiv \mathcal{I}_{\text {host }}(t=0)$, which reads

$$
\begin{aligned}
\mathcal{J}_{\text {host }} & =\left\langle\Phi_{n}\left|\mathcal{I}_{\text {host }}\right| \Phi_{n}\right\rangle \\
& =-\frac{i}{\hbar}\left\langle\Psi_{n}\left|\int_{-\infty}^{0}\left[\mathcal{I}_{\text {host }}, \tilde{\mathcal{H}}_{\text {tun }}(\tau)\right] d \tau\right| \Psi_{n}\right\rangle+\mathcal{O}\left(\tilde{\mathcal{H}}_{\text {tun }}^{2}\right),
\end{aligned}
$$

where we have regarded $\left\langle\Psi_{n}\left|\mathcal{I}_{\text {host }}\right| \Psi_{n}\right\rangle=0$ and by considering the thermal average on the latter equation, which gives

$$
\mathcal{J}_{\text {host }}=-\frac{i}{\hbar} \int_{-\infty}^{0} \operatorname{Tr}\left\{\varrho_{\varphi=0}\left[\mathcal{I}_{\text {host }}, \tilde{\mathcal{H}}_{\text {tun }}(\tau)\right]\right\} d \tau
$$

where $\varrho_{\varphi=0}$ is the density matrix of the system described by the Hamiltonian $\mathcal{H}_{\varphi=0}$ in Eq. (29). By applying the equationof-motion on $\mathcal{I}_{\text {host }}$, we show that

$$
\begin{aligned}
\mathcal{I}_{\text {host }}= & -\frac{i}{\hbar}\left[e \sum_{k} c_{k}^{\dagger} c_{k}, \mathcal{H}_{\varphi=0}\right] \\
= & \left(-\frac{i e}{\sqrt{2} \hbar}\right) V \sum_{k j}\left\{\left(c_{e k}^{\dagger} d_{j}-d_{j}^{\dagger} c_{e k}\right)+\left(c_{o k}^{\dagger} d_{j}-d_{j}^{\dagger} c_{o k}\right)\right\} \\
& +\left(-\frac{i e}{\hbar}\right) w \sum_{q \tilde{q}}\left(c_{o q}^{\dagger} c_{e \tilde{q}}-c_{e \tilde{q}}^{\dagger} c_{o q}\right),
\end{aligned}
$$

which, in combination with Eq. (35), leads to

$$
\mathcal{J}_{\text {host }}=-\frac{e}{\hbar} \Delta \mu \operatorname{Im} \int_{-\infty}^{+\infty} d \tau\left\{\sqrt{2} V \sum_{j} \mathcal{F}_{j}(-\tau)+2 w \mathcal{M}(-\tau)\right\}
$$

where

$$
\mathcal{F}_{j}(-\tau)=-\frac{i}{\hbar} \theta(-\tau) \operatorname{Tr}\left\{\varrho_{\varphi=0}\left[f_{o}^{\dagger} d_{j}, \sum_{q} c_{e q}^{\dagger}(\tau) c_{o q}(\tau)\right]\right\}
$$

and

$$
\mathcal{M}(-\tau)=-\frac{i}{\hbar} \theta(-\tau) \operatorname{Tr}\left\{\varrho_{\varphi=0}\left[f_{o}^{\dagger} f_{e}, \sum_{k} c_{e k}^{\dagger}(\tau) c_{o k}(\tau)\right]\right\}
$$

are retarded Green's functions, expressed in terms of the operators

$$
f_{o}=\sum_{\tilde{q}} c_{o \tilde{q}}
$$

and

$$
f_{e}=\sum_{q} c_{e q}
$$

In order to find a closed expression for the current $\mathcal{J}_{\text {host }}$, we should evaluate the integrals in the time coordinate $\tau$ of Eq. (37), which result in

$$
\begin{aligned}
\int_{-\infty}^{+\infty} d \tau \mathcal{F}_{j}(-\tau)= & \mathcal{Z}^{-1} \sum_{m n} \frac{\left(e^{-\beta E_{n}}-e^{-\beta E_{m}}\right)}{E_{n}-E_{m}+i \eta} \\
& \times\left\langle\Psi_{n}\left|f_{o}^{\dagger} d_{j}\right| \Psi_{m}\right\rangle\left\langle\Psi_{m}\left|\sum_{q} c_{e q}^{\dagger} c_{o q}\right| \Psi_{n}\right\rangle
\end{aligned}
$$

and

$$
\begin{aligned}
\int_{-\infty}^{+\infty} d \tau \mathcal{M}(-\tau)= & \mathcal{Z}^{-1} \sum_{m n} \frac{\left(e^{-\beta E_{n}}-e^{-\beta E_{m}}\right)}{E_{n}-E_{m}+i \eta} \\
& \times\left\langle\Psi_{n}\left|f_{o}^{\dagger} f_{e}\right| \Psi_{m}\right\rangle\left\langle\Psi_{m}\left|\sum_{q} c_{e q}^{\dagger} c_{o q}\right| \Psi_{n}\right\rangle
\end{aligned}
$$

where we have used $\mathcal{Z}$ as the partition function of $\mathcal{H}_{\varphi=0}\left|\Psi_{m}\right\rangle=E_{m}\left|\Psi_{m}\right\rangle, \mathcal{A}(\tau)=e^{\frac{i}{\hbar} \mathcal{H}_{\varphi=0} \tau} \mathcal{A} e^{-\frac{i}{\hbar} \mathcal{H}_{\varphi=0} \tau}$ for an arbitrary time-dependent operator $\mathcal{A}(\tau)$ and $\eta \rightarrow 0^{+}$. To eliminate the matrix element $\left\langle\Psi_{m}\left|c_{e q}^{\dagger} c_{o q}\right| \Psi_{n}\right\rangle$ in Eqs. (42) and (43), we calculate $\left\langle\Psi_{m}\left|\left[\sum_{q} c_{e q}^{\dagger} c_{o q}, \mathcal{H}_{\varphi=0}\right]\right| \Psi_{n}\right\rangle$, which gives

$$
\begin{aligned}
\left\langle\Psi_{m}\left|\sum_{q} c_{e q}^{\dagger} c_{o q}\right| \Psi_{n}\right\rangle= & -\frac{\sqrt{2} V}{\left(E_{n}-E_{m}\right)} \\
& \times \sum_{\tilde{j}}\left\langle\Psi_{m}\left|d_{\tilde{j}}^{\dagger} f_{o}\right| \Psi_{n}\right\rangle \\
& -\frac{2 w}{\left(E_{n}-E_{m}\right)}\left\langle\Psi_{m}\left|f_{e}^{\dagger} f_{o}\right| \Psi_{n}\right\rangle .
\end{aligned}
$$

By performing the substitutions of Eqs. (42), (43) with (44) in Eq. (37), we enclose the result into the function labeled by $\chi_{m n}$ to show that

$$
\begin{aligned}
\mathcal{J}_{\text {host }} & =\frac{e}{\hbar} \pi \Delta \mu \mathcal{Z}^{-1} \sum_{m n} \chi_{m n} \frac{\left(e^{-\beta E_{n}}-e^{-\beta E_{m}}\right)}{E_{n}-E_{m}} \delta\left(E_{n}-E_{m}\right) \\
& =-\frac{e}{\hbar} \pi \Delta \mu \beta \sum_{m n}\left[\mathcal{Z}^{-1} e^{-\beta E_{n}} \delta\left(E_{n}-E_{m}\right)\right] \chi_{n m},
\end{aligned}
$$

where we have defined

$$
\begin{aligned}
\chi_{n m}= & (\sqrt{2} V)^{2} \sum_{j \tilde{j}}\left\langle\Psi_{n}\left|f_{o}^{\dagger} d_{j}\right| \Psi_{m}\right\rangle\left\langle\Psi_{m}\left|d_{\tilde{j}}^{\dagger} f_{o}\right| \Psi_{n}\right\rangle \\
& +2 \sqrt{2} V(2 w) \sum_{j}\left\langle\Psi_{n}\left|f_{o}^{\dagger} d_{j}\right| \Psi_{m}\right\rangle\left\langle\Psi_{m}\left|f_{e}^{\dagger} f_{o}\right| \Psi_{n}\right\rangle \\
& +(2 w)^{2}\left\langle\Psi_{n}\left|f_{o}^{\dagger} f_{e}\right| \Psi_{m}\right\rangle\left\langle\Psi_{m}\left|f_{e}^{\dagger} f_{o}\right| \Psi_{n}\right\rangle .
\end{aligned}
$$

In this calculation, we have used

$$
\begin{aligned}
& \left\langle\Psi_{n}\left|f_{o}^{\dagger} d_{j}\right| \Psi_{m}\right\rangle\left\langle\Psi_{m}\left|f_{e}^{\dagger} f_{o}\right| \Psi_{n}\right\rangle \\
& \quad=\left\langle\Psi_{n}\left|f_{o}^{\dagger} f_{e}\right| \Psi_{m}\right\rangle\left\langle\Psi_{m}\left|d_{j}^{\dagger} f_{o}\right| \Psi_{n}\right\rangle,
\end{aligned}
$$

with

$$
\frac{\left(e^{-\beta E_{n}}-e^{-\beta E_{m}}\right)}{E_{n}-E_{m}}=-\beta e^{-\beta E_{n}}
$$

in the limit $E_{n} \rightarrow E_{m}$. The property $\left[\mathcal{H}_{e}, \mathcal{H}_{o}\right]=0$ ensures the partitions $E_{n}=E_{n}^{e}+E_{n}^{o}$ and $\mathcal{Z}=\mathcal{Z}_{e} \mathcal{Z}_{o}$ for the Hamiltonians 
$\mathcal{H}_{e}$ and $\mathcal{H}_{o}$, respectively in the brackets of Eq. (45), thus leading to

$$
\begin{aligned}
\mathcal{Z}^{-1} e^{-\beta E_{n}} \delta\left(E_{n}-E_{m}\right)= & \frac{1}{\beta} \mathcal{Z}_{e}^{-1} \mathcal{Z}_{o}^{-1} \int d \varepsilon\left(-\frac{\partial f_{F}}{\partial \varepsilon}\right) \\
& \times\left(e^{-\beta E_{n}^{e}}+e^{-\beta E_{m}^{e}}\right)\left(e^{-\beta E_{n}^{o}}+e^{-\beta E_{m}^{o}}\right) \\
& \times \delta\left(\varepsilon+E_{n}^{e}-E_{m}^{e}\right) \delta\left(\varepsilon+E_{n}^{o}-E_{m}^{o}\right) .
\end{aligned}
$$

Therefore, we substitute Eqs. (46) and (48) in Eq. (45) to calculate $\frac{\partial}{\partial \varphi} \mathcal{J}_{\text {host }}(\varphi=0)$. The comparison of such a result with Eq. (27) allows us to find

$$
\mathcal{T}_{\text {full }}(\varepsilon)=(2 \pi w)^{2} \tilde{\rho}_{\text {LDOS }}(\varepsilon) \tilde{\rho}_{\text {tip }}(\varepsilon),
$$

where

$$
\tilde{\rho}_{\text {LDOS }}(\varepsilon)=-\frac{1}{\pi} \operatorname{Im}\left(\tilde{\mathcal{R}}_{\psi_{e} \psi_{e}}\right)
$$

and

$$
\mathcal{R}_{\psi_{e} \psi_{e}}=-\frac{i}{\hbar} \theta(t) \operatorname{Tr}\left\{\varrho_{\mathrm{e}}\left[\psi_{e}(t), \psi_{e}^{\dagger}(0)\right]_{+}\right\},
$$

with the former as the renormalized LDOS of the "host+adatoms" system described by the Hamiltonian of Eq. (30), which is affected by the STM tip via the scattering term $w \sum_{k q} c_{e k}^{\dagger} c_{e q}$, thus leading to

$$
\psi_{e}=f_{e}+\left(\pi \Delta \rho_{0}\right)^{1 / 2} \gamma \sum_{j} d_{j}
$$

and

$$
\begin{aligned}
\tilde{\mathcal{R}}_{\psi_{e} \psi_{e}}= & \tilde{\mathcal{R}}_{f_{e} f_{e}}+\left(\pi \rho_{0} \Delta\right) \gamma^{2} \sum_{j l} \tilde{\mathcal{R}}_{d_{j} d_{l}} \\
& +2\left(\pi \rho_{0} \Delta\right)^{1 / 2} \gamma \sum_{j} \tilde{\mathcal{R}}_{d_{j} f_{e}}
\end{aligned}
$$

that generalize Eqs. (6) and (17), respectively, with a renormalized Anderson parameter

$$
\Delta=2 \pi V^{2} \rho_{0}
$$

and Fano factor

$$
\gamma=\left(\pi \rho_{0} \Delta\right)^{-1 / 2}\left(\frac{\sqrt{2} V}{2 w}\right) .
$$

Additionally, the scattering term $-w \sum_{k q} c_{o k}^{\dagger} c_{o q}$ renormalizes the DOS of the STM tip due to the Hamiltonian of Eq. (31), which provides

$$
\tilde{\rho}_{\text {tip }}(\varepsilon)=-\frac{1}{\pi} \operatorname{Im}\left(\tilde{\mathcal{R}}_{f_{a} f_{o}}\right) .
$$

From Eqs. (30) and (41), we make the substitutions $\mathcal{A}=\mathcal{B}=f_{e}$ and $\mathcal{H}_{i}=\mathcal{H}_{e}$ in Eq. (16), which gives

$$
\begin{aligned}
\tilde{\mathcal{R}}_{f_{e} f_{e}}= & \frac{\pi \rho_{0}(\bar{\gamma}-i)}{1-\sqrt{x}(\bar{\gamma}-i)}+\pi \rho_{0} \Delta\left[\frac{(\bar{\gamma}-i)}{1-\sqrt{x}(\bar{\gamma}-i)}\right]^{2} \\
& \times \sum_{j l} \tilde{\mathcal{R}}_{d_{j} d_{l}}(\varepsilon),
\end{aligned}
$$

where we have used the mixed Green's function

$$
\tilde{\mathcal{R}}_{d_{j} f_{e}}=\sqrt{\pi \Delta \rho_{0}} \frac{(\bar{\gamma}-i)}{1-\sqrt{x}(\bar{\gamma}-i)} \sum_{l} \tilde{\mathcal{R}}_{d_{j} d_{l}},
$$

determined from Eq. (16) by considering $\mathcal{A}=d_{j}, \mathcal{B}=f_{e}$ and $\mathcal{H}_{i}=\mathcal{H}_{e}$, with the parameter $x$ being the same as found in Eq. (26). We point out that, Eqs. (56) and (57) constitute respectively, generalizations of Eqs. (18) and (19), where the latter can be obtained from the former by making $x \ll 1$. Thus, the imaginary parts of Eqs. (56) and (57) for the wide band limit $D \rightarrow \infty$, become

$$
\begin{aligned}
\operatorname{Im}\left(\tilde{\mathcal{R}}_{f_{e} f_{e}}\right)= & -\frac{\pi \rho_{0}}{1+x}-\frac{(1-x)}{(1+x)^{2}} \pi \Delta \rho_{0} \sum_{j l} \operatorname{Im}\left(\tilde{\mathcal{R}}_{d_{j} d_{l}}\right) \\
& +\frac{2 \sqrt{x}}{(1+x)^{2}} \pi \Delta \rho_{0} \sum_{j l} \operatorname{Re}\left(\tilde{\mathcal{R}}_{d_{j} d_{l}}\right)
\end{aligned}
$$

and

$$
\begin{aligned}
\operatorname{Im}\left(\tilde{\mathcal{R}}_{d_{j} f_{e}}\right)= & -\frac{\sqrt{x \pi \Delta \rho_{0}}}{1+x} \sum_{l} \operatorname{Im}\left(\tilde{\mathcal{R}}_{d_{j} d_{l}}\right) \\
& -\frac{\sqrt{\pi \Delta \rho_{0}}}{1+x} \sum_{l} \operatorname{Re}\left(\tilde{\mathcal{R}}_{d_{j} d_{l}}\right),
\end{aligned}
$$

where we have used $\bar{\gamma} \rightarrow 0$. To conclude, we notice that $\tilde{\mathcal{R}}_{f_{a} f_{o}}$ is decoupled from the adatoms. Thereby, from Eqs. (31) and (40), we take $\mathcal{A}=\mathcal{B}=f_{o}$ and $\mathcal{H}_{i}=\mathcal{H}_{o}$ in Eq. (16) and we obtain

$$
\operatorname{Im}\left(\tilde{\mathcal{R}}_{f_{o} f_{o}}\right)=-\frac{\pi \rho_{0}}{1+x},
$$

which is equal to the first term of Eq. (58).

Thus the substitution of Eqs. (58)-(60) in Eq. (49), leads to

$$
\begin{aligned}
T_{\text {full }}(\varepsilon)= & \frac{\mathcal{T}_{\text {full }}(\varepsilon)}{\overline{\mathcal{T}}_{b}}=1+\bar{\Gamma} \sum_{j l}\left[\left(1-q_{b}^{2}\right) \operatorname{Im}\left(\tilde{\mathcal{R}}_{d_{j} d_{l}}\right)\right. \\
& \left.+2 q_{b} \operatorname{Re}\left(\tilde{\mathcal{R}}_{d_{j} d_{l}}\right)\right],
\end{aligned}
$$

where

$$
\overline{\mathcal{T}}_{b}=\frac{4 x}{(1+x)^{2}}
$$

represents the transmittance in the absence of the adatoms and MBSs (background contribution),

$$
\bar{\Gamma}=\frac{\Delta}{1+x}
$$


is an effective coupling and

$$
q_{b}=\frac{(1-x)}{2 \sqrt{x}}
$$

is the Fano parameter. Notice that Eq. (61) has the same form of Eq. (25), but with $q_{0}$ replaced by $q_{b}$. In this work, we will focus on the limit $q_{0}=q_{b}=0$.

\section{Green's functions of the adatoms}

In this section, we calculate the Green's functions $\tilde{\mathcal{R}}_{d_{j} d_{l}}$ within the wide band limit $D \rightarrow \infty$. We point out that, the expressions derived here describe the situation of Sec. II B 1 for an STM tip as probe with $\Gamma$ instead of $\Delta$ [see Eqs. (8) and (53)] and by assuming $x \ll 1$ [Eq. (26)], otherwise, they belong to the case of Sec. II B 2 . We begin by applying the equation-of-motion procedure on

$$
\mathcal{R}_{d_{j} d_{l}}=-\frac{i}{\hbar} \theta(t) \operatorname{Tr}\left\{\varrho_{\mathrm{s}}\left[d_{j}(t), d_{l}^{\dagger}(0)\right]_{+}\right\}
$$

where $\mathrm{s}=$ host + ads or $\mathrm{s}=\mathrm{e}$, and changing to the energy domain $\varepsilon$, we obtain the following relation:

$$
\left(\varepsilon-\tilde{\varepsilon}_{j}-i \Sigma^{I}-\delta_{j 1} \Sigma_{\mathrm{MBS} 1}\right) \tilde{\mathcal{R}}_{d_{j} d_{l}}=\delta_{j l}+\Sigma \sum_{\tilde{l} \neq j} \tilde{\mathcal{R}}_{d \tilde{l}} d_{l},
$$

with

$$
\tilde{\varepsilon}_{j}=\varepsilon_{j}+\Sigma^{R}
$$

is the adatom level renormalized by the STM tip-host coupling $w$, with $\Sigma=\Sigma^{R}+i \Sigma^{I}$,

$$
\begin{gathered}
\Sigma^{R}=-\frac{\sqrt{x}}{1+x} \Delta, \\
\Sigma^{I}=-\frac{\Delta}{1+x}
\end{gathered}
$$

and

$$
\Sigma_{\mathrm{MBS} 1}=\lambda^{2} K\left(1+\lambda^{2} \tilde{K}\right)
$$

as the self-energy due to the MBS 1 coupled to the adatom 1,

$$
K=\frac{1}{2}\left(\frac{1}{\varepsilon-\epsilon_{M}+i \eta}+\frac{1}{\varepsilon+\epsilon_{M}+i \eta}\right)
$$

and

$$
\tilde{K}=\frac{K}{\varepsilon+\tilde{\varepsilon}_{1}-i \Sigma^{I}-\lambda^{2} K}
$$

which have the same forms as found in Ref. 22. Thus, the solution of Eq. (66) provides

$$
\tilde{\mathcal{R}}_{d_{1} d_{1}}=\frac{1}{\varepsilon-\tilde{\varepsilon}_{1}-i \Sigma^{I}-\Sigma_{\mathrm{MBS} 1}-\mathcal{C}_{2}}
$$

as the Green's function of the adatom 1, with

$$
\mathcal{C}_{j}=\frac{\left(\Sigma^{R}+i \Sigma^{I}\right)^{2}}{\varepsilon-\tilde{\varepsilon}_{j}-i \Sigma^{I}}
$$

as the self-energy due to the presence of the $j$ th adatom. For $\mathcal{C}_{2}=0$, we highlight that Eq. (73) is reduced to the Green's function of the single QD system found in Ref. 22. In the case of the adatom 2, we have

$$
\tilde{\mathcal{R}}_{d_{2} d_{2}}=\frac{1-\tilde{\mathcal{R}}_{d_{1} d_{1}}^{0} \Sigma_{\mathrm{MBS} 1}}{\varepsilon-\tilde{\varepsilon}_{2}-i \Sigma^{I}-\frac{\tilde{\mathcal{R}}_{d_{1} d_{1}}^{0}}{\tilde{\mathcal{R}}_{d_{2} d_{2}}^{0}} \Sigma_{\mathrm{MBS} 1}-\mathcal{C}_{1}},
$$

where $\tilde{\mathcal{R}}_{d_{1} d_{1}}^{0}=1 /\left(\varepsilon-\tilde{\varepsilon}_{1}-i \Sigma^{I}\right)$ and $\tilde{\mathcal{R}}_{d_{2} d_{2}}^{0}=1 /\left(\varepsilon-\tilde{\varepsilon}_{2}-i \Sigma^{I}\right)$ represent the corresponding Green's functions for the single adatom system without Majoranas. To conclude, the mixed Green's functions are

$$
\tilde{\mathcal{R}}_{d_{2} d_{1}}=\frac{\Sigma^{R}+i \Sigma^{I}}{\varepsilon-\tilde{\varepsilon}_{2}-i \Sigma^{I}} \tilde{\mathcal{R}}_{d_{1} d_{1}}
$$

and

$$
\tilde{\mathcal{R}}_{d_{1} d_{2}}=\frac{\Sigma^{R}+i \Sigma^{I}}{\varepsilon-\tilde{\varepsilon}_{1}-i \Sigma^{I}-\Sigma_{\mathrm{MBS} 1}} \tilde{\mathcal{R}}_{d_{2} d_{2}} .
$$

The main result of this section is the emergence of a lack of symmetry in these Green's functions. This property lies on the coupling of the adatom 1 with the MBS 1 . To notice that, let us examine the situation where the adatom 1 is decoupled from the MBS 1, which can be obtained with $\Sigma_{\text {MBS1 }}=0$ in Eq. (70). By inspection of Eqs. (73) and (75)-(77), we verify that the functions $\tilde{\mathcal{R}}_{d_{1} d_{1}}$ and $\tilde{\mathcal{R}}_{d_{1} d_{2}}$ can be determined by the swap of the indexes $1 \leftrightarrow 2$ in $\tilde{\mathcal{R}}_{d_{2} d_{2}}$ and $\tilde{\mathcal{R}}_{d_{2} d_{1}}$, respectively. However, in the opposite situation with $\Sigma_{\mathrm{MBS} 1} \neq 0$, this symmetry is broken. Thus, in Sec. III, we will investigate this lack of symmetry via the transmittances of Eqs. (25) and (61). To this end, we will follow the two-stage procedure presented in Sec. I.

\section{RESULTS}

Here, we consider the Kitaev chain long enough, which forces $\epsilon_{M} \sim e^{-L / \xi} \rightarrow 0$ in Eq. (2) for $L \gg \xi$. We adopt typical values for adatoms in metals [Ref. 32]: $\Delta=\Gamma=0.2$ for the Anderson parameters of Eqs. (8) and (53), $\lambda, \varepsilon_{1}=-\frac{\Delta \varepsilon}{2}$, $\varepsilon_{2}=\frac{\Delta \varepsilon}{2}$, the symmetric detuning $\Delta \varepsilon=\varepsilon_{2}-\varepsilon_{1}$ and $\varepsilon$ in units of $\mathrm{eV}$.

In order to investigate the transmittance $T_{\text {full }}(\varepsilon)$ of Eq. (61) as a function of the single particle energy $\varepsilon$, in Fig. 2 we use $\lambda=5 \Delta$, with $x=1$ and Fano factor $q_{b}=0$ [Eq. (64)]. This set of parameters allows one to emulate the situation where the STM tip is strongly connected to the host surface and therefore, considered in the same footing as the "host + adatoms" system.

In Fig. 2(a) for the case of a free system, i.e., without MBSs [solid-green curve], we observe two antiresonances, each one placed around the corresponding adatom level given by $\varepsilon_{1}=-2.5$ and $\varepsilon_{2}=+2.5$, respectively. We name 

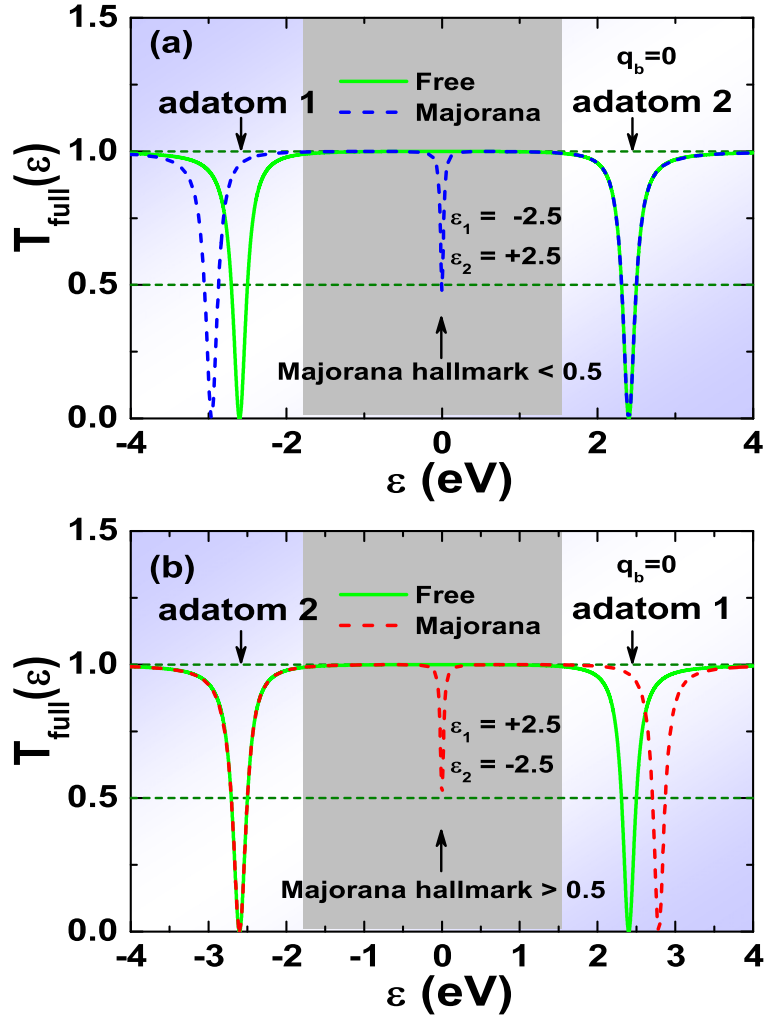

FIG. 2. Parameters employed: $\epsilon_{M}=0$ [long enough Kitaev chain], $\lambda=5 \Delta$ and $\Delta=0.2$ [see Eqs. (2) and (53)]. Transmittance $T_{\text {full }}(\varepsilon)$ determined by Eq. (61) in the Fano regime $q_{b}=0$ [Eq. (64)] as a function of the single particle energy $\varepsilon$. In the panels (a) and (b) we have: the solid-green lineshape is for the apparatus of Fig. 1 in the absence of the MBS 1. Implementation of the two-stage procedure of Sec. I: (a) $\varepsilon_{1}=-2.5$ and $\varepsilon_{2}=+2.5$ : The dashed-blue curve corresponds to the system coupled to the MBS 1. (b) $\varepsilon_{1}=+2.5$ and $\varepsilon_{2}=-2.5$ : The dashed-red lineshape is for the MBS 1 . Here we see the main result of this procedure: the formation of a Majorana dip with an amplitude that fluctuates slightly around $1 / 2$, but it remains pinned at zero-bias even by performing the gates swap. The satellite dips do not share such a feature, they become significantly shifted under the permutation of the levels in the adatoms.

these antiresonances as satellite dips. Off the antiresonances, the transmittance approaches the unitary limit and the conductance reaches $\mathcal{G}=\mathcal{G}_{0}=e^{2} / h$. Notice, for instance, the central region bounded by the range $-1.75 \lessgtr e \varphi \lesssim 1.5$ [shaded region], where we have a ballistic plateau with the aforementioned conductance. In this free system, the Green's functions of the model are symmetric under the permutation of the indexes that designate the parameters of the adatoms. This property is confirmed by the corresponding solid-green curve of Fig. 2(b), obtained with $\varepsilon_{1}=+2.5$ and $\varepsilon_{2}=-2.5$, which agrees with that for $\varepsilon_{1}=-2.5$ and $\varepsilon_{2}=+2.5$ in Fig. 2(a). Therefore, the two-stage procedure proposed in this work, in particular for the case of a free double adatom system, yields two identical curves for the transmittance. However, for the device side-coupled to the MBS 1 , a novel feature emerges in the central region.

By fixing $\varepsilon_{1}=-2.5$ and $\varepsilon_{2}=+2.5$, a dip of amplitude nearby $1 / 2$ arises in the middle of the ballistic plateau, due to the MBS 1 attached to the adatom 1 [see the dashed-blue curve in Fig. 2(a)]. For this situation, the dip around $\varepsilon_{2}=+2.5$ coincides with the corresponding one found in the solid-green curve of the free setup, which is due to the adatom 2 decoupled from the MBS 1. Moreover, the antiresonance in the vicinity of $\varepsilon_{1}=-2.5$ is not coincident with that in Fig. 2(a) of the solid-green curve for the free system. As we can see, the position of such an antiresonance is shifted as the aftermath of the coupling between the MBS 1 and the adatom 1. After the swap procedure, which leads to $\varepsilon_{1}=+2.5$ and $\varepsilon_{2}=-2.5$, the satellite dips of Fig. 2(b) [see the dashed-red lineshape] become reversed with respect to those found in Fig. 2(a).

We emphasize that the central antiresonance remains placed at zero-bias, but its amplitude fluctuates slightly around 1/2. This behavior of the central dip appears in Fig. 3 (a), which can be clearly visualized in the dashed-blue and red lineshapes, respectively. Therefore, a pinned antiresonance protected against the two-stage procedure emerges in the transmittance, which is placed at the zero-bias and characterized by an amplitude nearby $1 / 2$. In contrast, the satellite dips do not share such a pinning, since they move significantly under the permutation of the levels in the adatoms. However, the complete robustness of the Majorana hallmark does not exist anymore as found in Refs. 22 and 23: the amplitude is not fixed at $1 / 2$ as a straight result of the interplay between the adatom decoupled from the Kitaev
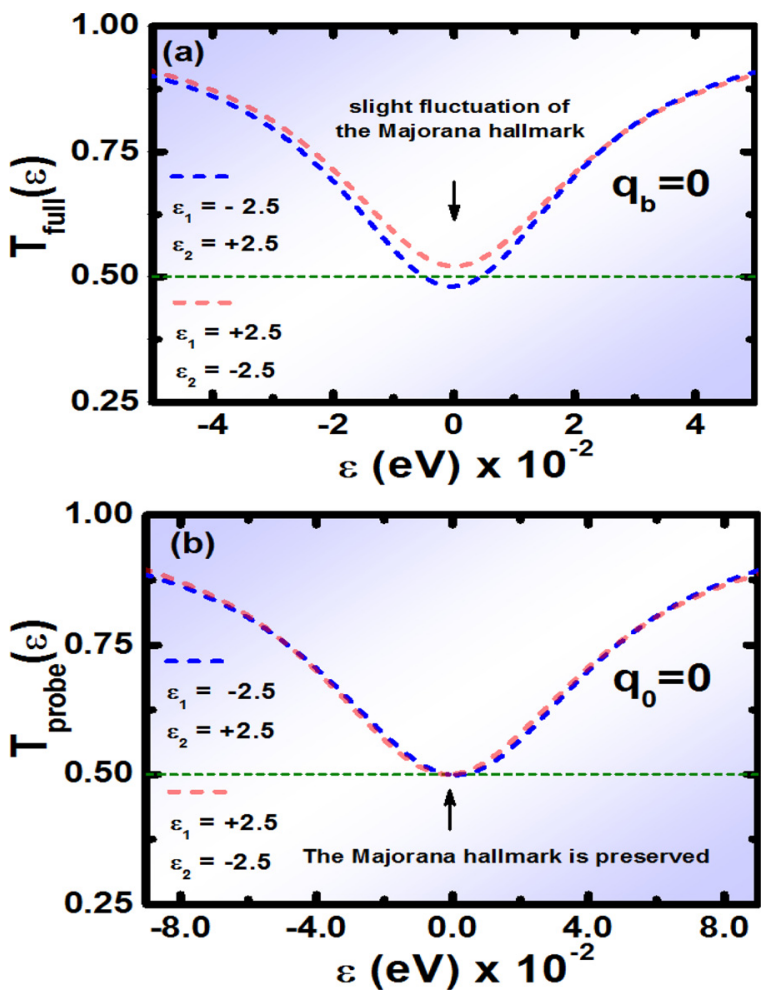

FIG. 3. Parameters employed: $\epsilon_{M}=0$ [long enough Kitaev chain], $\lambda=5 \Delta$ and $\Delta=\Gamma=0.2$ [see Eqs. (2), (8), and (53)]. Transmittance as a function of the single particle energy $\varepsilon$. Implementation of the two-stage procedure of Sec. I: (a) via the transmittance $T_{\text {full }}(\varepsilon)$ of Eq. (61) in the Fano regime $q_{b}=0$ [Eq. (64)], where we see the formation of a Majorana dip with an amplitude that fluctuates slightly around 1/2 (Majorana hallmark), but it remains pinned at zero-bias even by performing the gates swap. In panel (b), the transmittance $T_{\text {probe }}(\varepsilon)$ of Eq. (25) for $q_{0}=0$ does not exhibit such a fluctuation. The Majorana hallmark remains unchanged for the STM tip considered as a probe. 
chain and the Fano regime as well, obtained with $x=1$ in Eq. (64). In this situation, the real and imaginary parts of the self-energy $\Sigma$, which read $\Sigma^{R}$ and $\Sigma^{I}$, respectively, given by Eqs. (68) and (69), depend on $x$. Otherwise, it would correspond to the case of the tip considered as a probe of the LDOS for the "host+adatoms" system, which suppresses the fluctuation of the Majorana hallmark. This feature can be observed by using the transmittance $T_{\text {probe }}(\varepsilon)$ of Eq. (25) with $q_{0}=0$, which is confirmed by the dashed-blue and red lineshapes of Fig. 3(b). In fact, it can be observed an antiresonance pinned at zero-bias characterized by a constant amplitude of $1 / 2$. In this case, $\Sigma^{R}$ and $\Sigma^{I}$ do not depend on $x$, since $x \ll 1$ for a probe tip [see Eq. (26)]. As a result, the Majorana hallmark is preserved under the gates swap.

Thus in order to explore the effects due to the fluctuation of the zero-bias transmittance, we present the analysis of $T_{\text {full }}(0)$ and $T_{\text {probe }}(0)$ as a function of the symmetric detuning $\Delta \varepsilon$. In both cases, the Fano parameters are $q_{b}=0$ and $q_{0}=0$, which according to Fano's theory, lead to a destructive interference pattern. Such a behavior can be seen in the transmittance versus $\varepsilon$ plots of Figs. 3(a) and 3(b). Additionally, we point out that the Majorana dip verified in
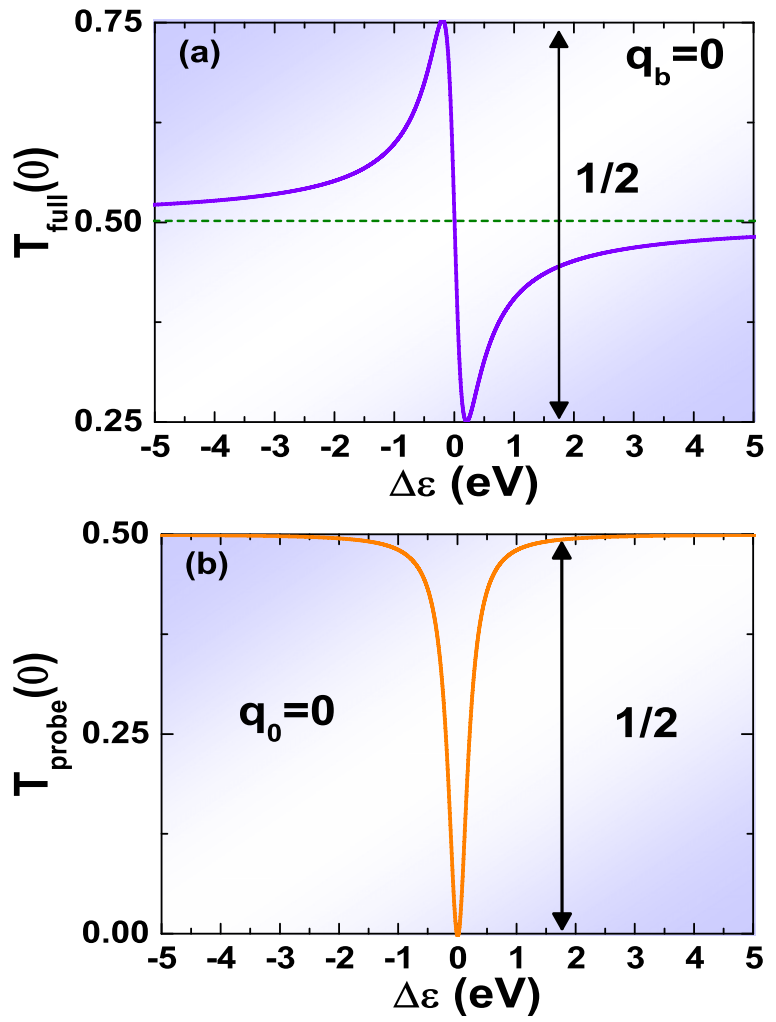

FIG. 4. Parameters employed: $\epsilon_{M}=0$ [long enough Kitaev chain], $\lambda=5 \Delta$ and $\Delta=\Gamma=0.2$ [see Eqs. (2), (8), and (53)]. Panel (a): Transmittance $T_{\text {full }}$ (0) of Eq. (61) in the Fano regime $q_{b}=0$ [Eq. (64)] as a function of the symmetric detuning $\Delta \varepsilon=\varepsilon_{2}-\varepsilon_{1}$. For the STM tip in the same footing as the "host+adatoms" system, we see a novel feature in the transmittance profile: an unexpected Fano lineshape emerges and the Fano dip is not verified. Pained (b): in the case of the STM tip as a probe, the transmittance $T_{\text {probe }}(0)$ of Eq. (25) with $q_{0}=0$ leads to the standard Fano antiresonance. We remark that despite the small difference in the Majorana dip of Fig. 3(a) with respect to that found in Fig. 3(b), the zero-bias transmittance as a function of the detuning $\Delta \varepsilon$, yields two distinct lineshapes. However, in both situations, the transmittance does not exceed an amplitude of $1 / 2$. the former differs slightly with respect to that found in the latter. Remarkably, the slight fluctuation of the Majorana hallmark in Fig. 3(a) is able to provide an unexpected profile of $T_{\text {full }}(0)$ versus $\Delta \varepsilon$, which differs expressively of a Fano dip. The result of this analysis appears in the solid-violet curve of Fig. 4(a), where it is observed that the transmittance approaches $1 / 2$ from upper (lower) values for $\Delta \varepsilon<0$ $(\Delta \varepsilon>0)$. In the domain of $\Delta \varepsilon<0$, it reaches the maximum value of $3 / 4$, while for $\Delta \varepsilon>0$, it decreases to $1 / 4$. Notice that the variation of the transmittance with $\Delta \varepsilon$ does not exceed an amplitude of $1 / 2$ and particularly at $\Delta \varepsilon=0$, the transmittance recovers the Majorana hallmark 1/2. On the other hand, in Fig. 4(b), the transmittance $T_{\text {probe }}(0)$ as a function of $\Delta \varepsilon$ in the solid-orange curve, displays the standard profile of Fano antiresonance for $q_{0}=0$. Notice that in both Figs. 4(a) and 4(b), the variation of the transmittance with $\Delta \varepsilon$ is $1 / 2$. We highlight that the unexpected Fano profile found in this work becomes a way to identify the existence of isolated MBSs, since the lineshape in Fig. 4(a) is due to a long enough Kitaev chain within the topological phase.

In summary, despite the same Fano parameters $q_{0}=0$ and $q_{b}=0$ in $T_{\text {probe }}(0)$ and $T_{\text {full }}(0)$, respectively, for Eqs. (25) and (61), which lead to Fano dips slightly different as those found in Figs. 3(a) and 3(b), we demonstrate in this

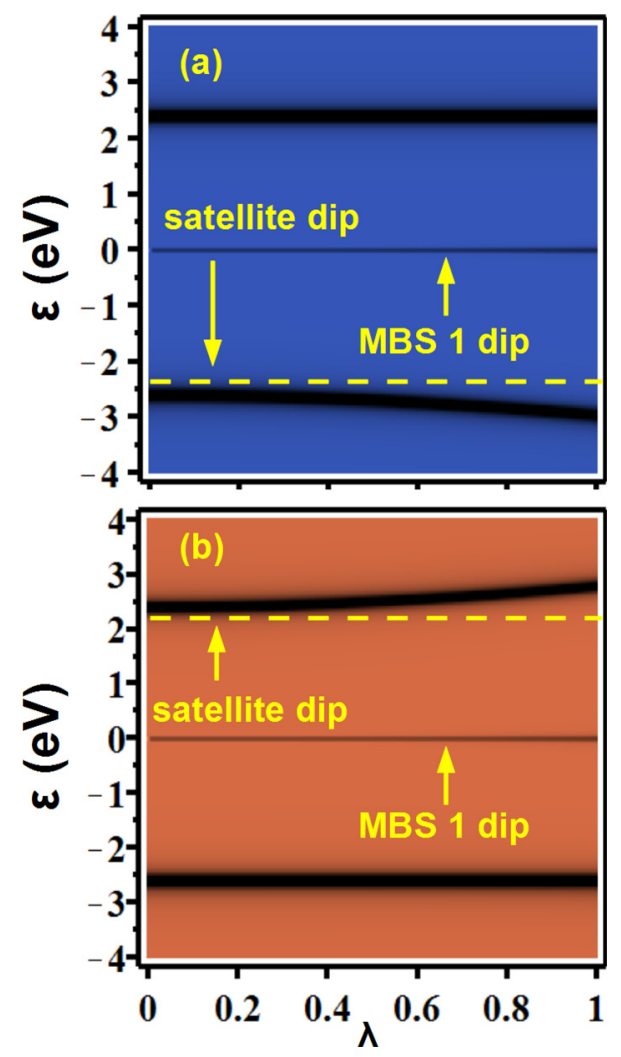

FIG. 5. Parameters employed: $\epsilon_{M}=0$ [long enough Kitaev chain], $\Delta=0.2$ [see Eqs. (2) and (53)]. Density plots of the transmittance $T_{\text {full }}(\varepsilon)$ determined by Eq. (61) in the Fano regime $q_{b}=0$ [Eq. (64)] as a function of the single particle energy $\varepsilon$ and the coupling $\lambda$ in units of $\Delta$. Implementation of the two-stage procedure of Sec. I: (a) $\Delta \varepsilon=+5$ (b) $\Delta \varepsilon=-5$. Here we see the main result of this procedure: the formation of a Majorana dip (in black) pinned at zero-bias even by performing the gates swap. The satellite dips (also in black) do not share such a feature being significantly shifted under the permutation of the levels in the adatoms. 
work that the usual hypothesis of the STM tip acting as a probe is insensitive for the complete knowing of the zero-bias transmittance versus the symmetric detuning $\Delta \varepsilon$. To overcome such an obstacle, the proper description should consider the STM tip in the same footing as the "host + adatoms" system. It is worth mentioning that we do not present the results for the case $q_{b} \gg 1$, since it still obeys the standard Fano's theory, which gives a resonance profile in the $T_{\text {full }}(0)$ versus $\Delta \varepsilon$ plot as expected. In Fig. 5 the density plots for $T_{\text {full }}(\varepsilon)$ of Eq. (61) with $q_{b}=0$ as a function of $\varepsilon$ and the coupling $\lambda$ are shown. In these graphs, dips appear (black color regions) being possible to observe that the MBS 1 dip at zero-bias is the only structure that does not change with the implementation of the two-stage procedure as well as with the increase of $\lambda$. On the other hand, the positions of the satellite dips are displaced by changing $\lambda$ and no pinning is observed. This feature can be visualized in the dips that deviate from the yellow-dashed lines in Figs. 5(a) and 5(b), respectively, for $\Delta \varepsilon=+5$ and $\Delta \varepsilon=-5$.

\section{CONCLUSIONS}

We have explored theoretically in the context of quantum transport an effective Hamiltonian supporting Majorana quasiparticles for a long enough Kitaev chain in the topological phase. This system is coupled to a setup made by an STM tip and a metallic host with two adatoms. Our analysis has revealed that the Green's functions of the adatoms become symmetric by neglecting the hopping term between one adatom and a side-coupled MBS. However, if we consider this parameter relevant, a lack of symmetry manifests in these functions.

To read out this feature experimentally, it has been proposed a two-stage procedure of gates swap by using AFM tips. As a result, a persistent zero-bias dip with an amplitude nearby $1 / 2$ emerges in the transmittance arising from the isolated MBS under the aforementioned procedure. We have also verified that the fluctuation of the Majorana hallmark occurs only for the STM tip treated in the same footing as the "host+adatoms" system. In the case of an STM tip as a probe, the robustness of the Majorana hallmark is kept. However, this small difference between these two Majorana dips results in contrasting Fano profiles for the zero-bias transmittance versus the symmetric detuning. In the case of the STM tip acting as probe, Fano's theory is confirmed, but with the tip in the same footing as the "host+adatoms" system, an unexpected Fano lineshape appears. We conclude that to access this non trivial Fano profile, the assumption of an STM tip acting as a probe should not be used.

\section{ACKNOWLEDGMENTS}

The authors thank Drs. E. Vernek and J. C. Egues for valuable discussions. This work was supported by the Brazilian agencies CNPq, CAPES and PROPe/UNESP.

${ }^{1}$ J. Alicea, Rep. Prog. Phys. 75, 076501 (2012).

${ }^{2}$ M. Leijnse and K. Flensberg, Phys. Rev. B 84, 140501(R) (2011).

${ }^{3}$ H.-F. Lü, H.-Z. Lu, and S.-Q. Shen, Phys. Rev. B 86, 075318 (2012).

${ }^{4}$ M. Leijnse and K. Flensberg, Phys. Rev. B 86, 134528 (2012).

${ }^{5}$ K. Flensberg, Phys. Rev. Lett. 106, 090503 (2011).

${ }^{6}$ M. Leijnse and K. Flensberg, Phys. Rev. Lett. 107, 210502 (2011).

${ }^{7}$ A. Y. Kitaev, Phys. Usp. 44, 131 (2001).

${ }^{8}$ Y. Cao, P. Wang, G. Xiong, M. Gong, and X.-Q. Li, Phys. Rev. B 86, 115311 (2012).

${ }^{9}$ M. Gibertini, F. Taddei, M. Polini, and R. Fazio, Phys. Rev. B 85, 144525 (2012).

${ }^{10}$ L.-J. Lang and S. Chen, Phys. Rev. B 86, 205135 (2012).

${ }^{11}$ C.-H. Lin, J. D. Sau, and S. Das Sarma, Phys. Rev. B 86, 224511 (2012).

${ }^{12}$ X.-J. Liu and A. M. Lobos, Phys. Rev. B 87, 060504(R) (2013).

${ }^{13}$ D. Sticlet, C. Bena, and P. Simon, Phys. Rev. B 87, 104509 (2013).

${ }^{14}$ J. Liu, A. C. Potter, K. T. Law, and P. A. Lee, Phys. Rev. Lett. 109, 267002 (2012).

${ }^{15}$ S. Nakosai, J. C. Budich, Y. Tanaka, B. Trauzettel, and N. Nagaosa, Phys. Rev. Lett. 110, 117002 (2013).

${ }^{16}$ D. Roy, C. J. Bolech, and N. Shah, Phys. Rev. B 86, 094503 (2012).

${ }^{17}$ G. Moore and N. Read, Nucl. Phys. 360, 362 (1991).

${ }^{18}$ L. Fu, C. L. Kane, and E. J. Mele, Phys. Rev. Lett. 98, 106803 (2007).

${ }^{19}$ L. Fu and C. L. Kane, Phys. Rev. Lett. 100, 096407 (2008).

${ }^{20}$ J. D. Sau, R. M. Lutchyn, S. Tewari, and S. Das Sarma, Phys. Rev. Lett. 104, 040502 (2010).

${ }^{21}$ C. J. Bolech and E. Demler, Phys. Rev. Lett. 98, 237002 (2007).

${ }^{22}$ D. E. Liu and H. U. Baranger, Phys. Rev. B 84, 201308(R) (2011).

${ }^{23}$ E. Vernek, P. H. Penteado, A. C. Seridonio, and J. C. Egues, e-print arXiv: 1308.0092v2 [cond-mat.mes-hall] (2013).

${ }^{24}$ V. Mourik, K. Zuo, S. M. Frolov, S. R. Plissard, E. P. A. M. Bakkers, and L. P. Kouwenhoven, Science 336, 1003 (2012).

${ }^{25}$ A. Das, Y. Ronen, Y. Most, Y. Oreg, M. Heiblum, and H. Shtrikman, Nature Phys. 8, 887 (2012).

${ }^{26}$ A. C. Hewson, The Kondo Problem to Heavy Fermions (Cambridge University Press, Cambridge, England 1993).

${ }^{27}$ D. Goldhaber-Gordon, H. Shtrikman, D. Mahalu, D. Abusch- Magder, U. Meirav, and M. A. Kastner, Nature 391, 156 (1998).

${ }^{28}$ S. M. Cronenwett, T. H. Oosterkamp, and L. P. Kouwenhoven, Science 281, 540 (1998).

${ }^{29}$ A. F. Otte, M. Ternes, K. V. Bergmann, S. Loth, H. Brune, C. P. Lutz, C. F. Hirjibehedin, and A. J. Heinrich, Nature Phys. 4, 847 (2008).

${ }^{30}$ V. Madhavan, W. Chen, T. Jamneala, and F. Crommie, Phys. Rev. B 64, 165412 (2001).

${ }^{31}$ N. Knorr, M. A. Schneider, L. Diekhöner, P. Wahl, and K. Kern, Phys. Rev. Lett. 88, 096804 (2002).

${ }^{32}$ C. Y. Lin, A. H. C. Neto, and B. A. Jones, Phys. Rev. Lett. 97, 156102 (2006).

${ }^{33}$ M. Ternes, A. J. Heinrich, and W. D. Schneider, J. Phys.: Condens. Matter 21, 053001 (2009).

${ }^{34}$ U. Fano, Phys. Rev. 124, 1866 (1961).

${ }^{35}$ A. E. Miroshnichenko, S. Flach, and Y. S. Kivshar, Rev. Mod. Phys. 82, 2257 (2010).

${ }^{36}$ A. C. Seridonio, E. C. Siqueira, F. M. Souza, R. S. Machado, S. S. Lyra, and I. A. Shelykh, Phys. Rev. B 88, 195122 (2013).

${ }^{37}$ A. C. Seridonio, F. S. Orahcio, F. M. Souza, and M. S. Figueira, Phys. Rev. B 85, 165109 (2012). 